\title{
Visualizing Cancer Cell Dynamics in a Liver Slice Model
}

Priyodarshan Goswamee*, Sasi Arunachalam*, Riaz Nasim**, Marthe Howard* and David R. Giovannucci*

*Dept. of Neuroscience, University of Toledo Medical Center, Toledo, $\mathrm{OH}$

**Khyber Medical College, Peshawar, Pakistan

Many cancers, including midgut neuroendocrine cancers called carcinoid, typically metastasize to the liver [1]. However, cancer cell interactions with the liver tissue microenvironment are poorly understood.

To begin to address this problem, we developed an organ slice culture system that more closely resembles the three-dimensional, multicellular liver microenvironment than does a dispersed cell culture system. Using this ex-vivo system in combination with live-cell imaging methods and multiphoton microscopy we characterized complex spatial and temporal properties of cancer cell dynamics within a reduced tumor microenvironment.

In these experiments, human carcinoid cancer cells, stably transfected with GFP were introduced to the liver via hepatic portal vein injection. A long-lasting vital dye was also introduced to mark the vasculature. The liver was sectioned into $200 \mu \mathrm{m}$ thick slices and maintained in culture for up to two weeks and cancer cell dynamics were assessed by immunofluorescence methods or by timelapse imaging.

The introduced cancer cells had a proliferative index of about $50 \%$ that was not significantly different from cells grown on 2D substrates and remained relatively constant throughout the experiment. The cancer cells exhibited a variety of characteristic movements and readily interconverted between elongated and rounded (blebbing) modes of locomotion [2]. An addtional rapid form of movement was observed after several days in culture. This rapid form of movement appeared to correlate with tumor cell aggregation in slices. We also observed the formation of tumor-like structures ("tumorlets") following several days in culture.

The ability to form "tumorlets" appeared to require the prior perfusion of the portal vasculature with physiological saline, suggesting that resident immune cells both interfere and facilitate tumorogenesis. This indicates possible interaction of the cancer cells with the immune cells resident in the liver and/or present in the blood $[3,4]$.

This ex vivo model provides a tractable and cost efficient accompaniment to intravital microscopy for studying tumor formation in mouse liver. High resolution confocal imaging of the cancer cells revealed multiple distinct types of cell motilities and indicated that tumor cell aggregation may play a significant role in carcinoid tumor formation in the liver. Some of these properties were only observed after several days in organ slice culture suggesting important interactions with resident immune or other endogenous cells in the liver. 


\section{References}

○ [1] Semelka RC, et. al., J Magn Reson Imaging. 2000;11:141Y148.

○ [2] Fackler OT, et. al., J Cell Biol. 2008 Jun 16;181(6):879-84.

○ [3] Chouaib S, et. al., Crit Rev Immunol. 2010;30(6):529-45.

- [4] Sansone P, et. al., Curr Opin Genet Dev. 2011 Feb;21(1):80-5.

- This Research was supported by the Raymond and Beverly Sackler Foundation for Neuroendocrine Tumor Research 